\title{
Probiotics for treatment of nonalcoholic fatty liver disease: It is worth a try
}

\author{
Tian-Yi Ren', Xiao-Yan Li ${ }^{2}$, and Jian-Gao Fan ${ }^{1}$ \\ 'Department of Gastroenterology, Xinhua Hospital Affiliated to Shanghai Jiao Tong University School of Medicine, Shanghai Key Lab of \\ Pediatric Gastroenterology and Nutrition, Shanghai; ${ }^{2}$ Department of Oncology, the First Affiliated Hospital, Institute for Liver Diseases \\ of Anhui Medical University, Hefei, China
}

Keywords: Probiotics; Fatty liver disease; Metabolic syndrome; Therapy

\section{See Article on Page 110}

Nonalcoholic fatty liver disease (NAFLD), the metabolic stressinduced chronic hepatic injury, represents the prominent cause of liver disease nowadays and the anticipated health burden is huge in the world.' NAFLD encompasses a wide spectrum of liver histopathology, ranging from simple hepatic steatosis to nonalcoholic steatohepatitis (NASH), NASH-related cirrhosis, and hepatocellular carcinoma. ${ }^{2}$ To date, no drug has received U.S. Food and Drug Administration approval for NASH treatment, which would make NAFLD the most frequent indication for liver transplantation in the near future, given the global pandemic of obesity and type 2 diabetes. $^{3}$

Along with the escalating morbidity and mortality over the 2 decades, numerous studies have been conducted to explore the underlying mechanisms of NAFLD, which involve complex and multifactorial drivers of pathology. In brief, excess energy intake and impaired metabolic capacity facilitate the overload of free fatty acids in hepatocytes, which contributes to steatosis and lipotoxicity, cell injury and subsequent events including oxidative stress, endoplasmic reticulum stress, and inflammatory cascade reactions, etc. Besides the intrahepatic pathogenesis, dysfunctions of the cross-talk among central nervous system, adipose tissue and gut, due to either genetic predisposition or metabolic challenges, have also been proposed participating in NAFLD pathogenesis. ${ }^{4,5}$ Particularly, gut-liver axis has become a high-profile topic in the field of NAFLD research.

As there are over 100 trillion and 160 different species of microbes inhabiting in human gastrointestinal lumen, commensal bacteria significantly exceed human body in both cell number and gene abundance, and inevitably interact with host biological and metabolic functions. ${ }^{6}$ Although the exact mechanisms underlying gut flora function remain obscure, current evidence already suggests its unique roles in processing indigestive dietary contents, detoxifying drugs and preserving intestinal barrier. Perturbation of gut microbiota, i.e., gut dysbiosis, could serve as both a major cause and an important consequence of NAFLD development,

\section{Abbreviations: \\ ALT, alanine aminotransferase; AST, aspartate aminotransferase; BCAA, branched- chain amino acids; FXR, farnesoid X receptor; MAFLD, metabolic associated fatty liver disease; NAFLD, nonalcoholic fatty liver disease; NASH, nonalcoholic steatohepatitis; NF-KB, nuclear factor kappa B; PDFF, proton density fat fraction; PPARa, proliferators-activated receptor a; SCFAs, short-chain fatty acids; SREBP- $1 c$, sterol-regulatory element binding protein 1c}

\section{Corresponding author : Jian-Gao Fan}

Department of Gastroenterology, Xinhua Hospital Affiliated to Shanghai Jiao Tong University School of Medicine; Shanghai Key Lab of Pediatric Gastroenterology and Nutrition, 1665 Kong Jiang Road, Shanghai 200092, China

Tel: +86-21-25077340, Fax: +86-21-25077340

E-mail:fanjiangao@xinhuamed.com.cn https://orcid.org/0000-0002-8618-6402 


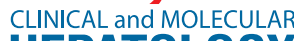

\section{HEPATOLOGY}

which hence persistently promotes hepatic dysfunction and injury in a cycle. The impaired intestinal integrity, bacterial translocation and endotoxin release could activate hepatic immune responses and induce liver injury stepwise. Disturbed intestinal microbiome also portends the loss of metabolite homeostasis, characterized by the dysregulations of bile acids, short-chain fatty acids (SCFAs), branched-chain amino acids (BCAA), choline, and endogenous ethanol, which could all contribute to NAFLD development. ${ }^{7}$ Putative mechanisms aiming to explain the roles of gut dysbiosis in NAFLD progression are outlined and discussed in Figure 1. So many pathogenic factors and loops are intertwined together, which renders the single-mechanism therapeutic approach barely feasible. Therefore, to develop therapeutic approaches specifically targeting the conditioning of gut microbiota is perfectly sensible.

In preclinical studies, a number of probiotics, such as Bifidobacterium, Lactobacillus, or Bacillus, have shown beneficial effects in rodent models of NAFLD. ${ }^{8-10}$ Daily administration of probiotics could reverse dysbiosis and substantially attenuate NAFLD features of animal by sensitizing insulin action, suppressing inflammation, and reducing lipogenesis. Several critical signaling pathways related to NAFLD pathogenesis, including sterol-regulatory element binding protein 1c (SREBP-1c), peroxisome proliferatorsactivated receptor a (PPARa), nuclear factor kappa B (NF-KB), and farnesoid $X$ receptor $(F X R)$, have been speculated as the effect targets of probiotics. Meanwhile, preliminary clinical researches on probiotics conducted so far have also yielded positive results in NAFLD patients. In the past 10 years, dozens of randomized controlled trials have been carried out to compare probiotics, including Bifidobacterium, Lactobacillus, Bacillus Enterococcus, Streptococcus thermophilus, Pediococcus pentosaceus, Propionibacterium, and Acetobacter with placebo in NAFLD patients, ${ }^{11}$ but they are still confined to tentatively exploration, considering the relatively small scales, arbitrary dosages and omnifarious observational indexes/endpoints. As reported, probiotics could improve

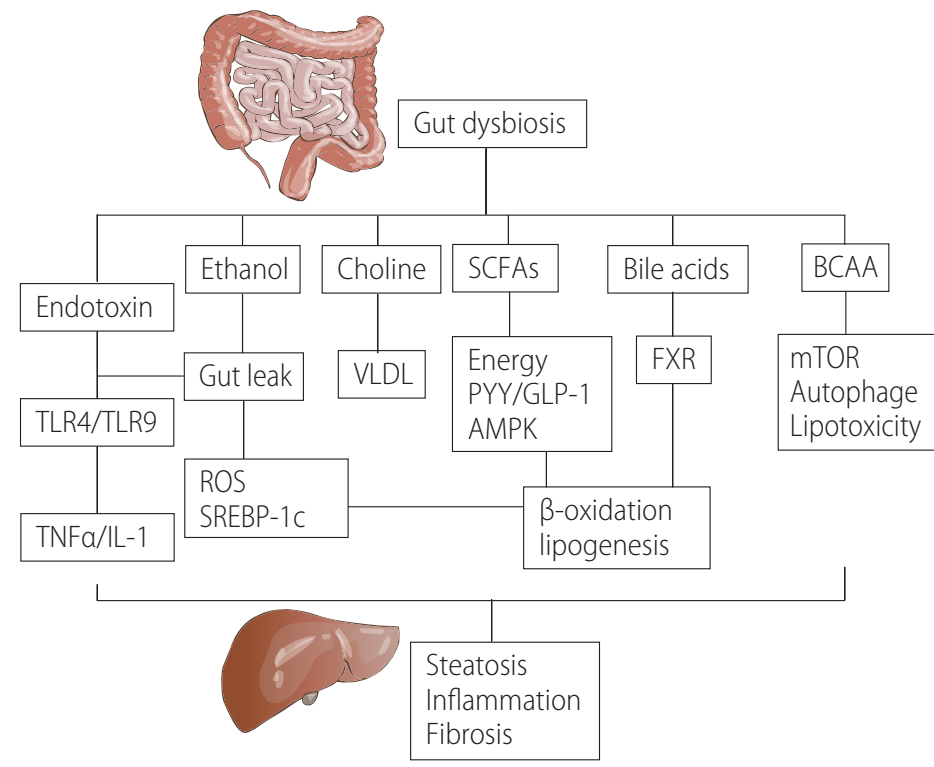

Figure 1. Key mechanisms for gut dysbiosis in NAFLD pathogenesis. Gut dysbiosis and impaired intestinal integrity lead to the release of endotoxin, which could activate TLR4/TLR9 and induce the secretion of proinflammatory cytokines such as TNFa and IL-1. Increased production of endogenous ethanol from food fermentation by dysregulated bacteria could damage the tight junctions of intestine wall and exacerbate gut leak. Moreover, ethanol is able to lay direct effects on hepatic steatosis and inflammation via activation of SREBP-1c and ROS production. Reduced conversion of choline during gut dysbiosis could inhibit VLDL function. As the metabolites of gut flora, SCFAs not only act on gut hormone PYY and GLP-1, but also provide excess energy and inhibit AMPK signaling in the liver. Consequently, lipogenesis is increased while $\beta$-oxidation is suppressed. Additionally, gut dysbiosis also interferes with bile acid metabolism and FXR, which indirectly contributes to the dysregulation of lipid metabolism. Another type of metabolite influenced by gut dysbiosis, BCAA, could exacerbate hepatic lipotoxicity and repress autophagy of hepatocyte via mTOR-activated signaling. All above-mentioned signaling pathways could synergistically promote development of hepatic steatosis, inflammation and fibrosis. SCFAs, short-chain fatty acids; BCAA, branched-chain amino acids; TLR, toll-like receoptor; VLDL, very low-density lipoprotein; PYY, peptide YY; GLP-1, glucagon-like peptide 1; AMPK, adenosine monophosphate activated protein kinase; FXR, farnesoid X receptor; mTOR, mammalian target of rapamycin; TNF, tumor necrotic factor; IL, interleukin; ROS, reactive oxygen species; SREBP-1C, sterol-regulatory element binding protein 1c; NAFLD, nonalcoholic fatty liver disease. 
serum aspartate aminotransferase (AST) and alanine aminotransferase (ALT) in both adult and pediatric NAFLD patients, ${ }^{12,13}$ and synergistically work with metformin to restore liver functions of NASH individuals. ${ }^{14}$ However, a trial in 2019 indicated that 12week treatment of multiple-strain probiotics in NAFLD patients improved only hepatic steatosis (identified by proton density fat fraction [PDFF] on magnetic resonance imaging), but not liver enzymes. ${ }^{15}$ These studies have provided necessary data regarding the efficacy and safety profiles of probiotics in clinical practice, and paved the way for more elaborate probiotic pharmacotherapies in the future.

In the current issue, Lee et al. ${ }^{16}$ described the Lactobacillus family abundance of gut microbiome in NAFLD patients compared with healthy controls, and further explored the medicinal potentials of four Lactobacillus strains in a diet-induced mouse of NAFLD. Compared with healthy subjects, NAFLD patients showed a significantly elevated Firmicutes/Bacteroidetes ratio, as well as reduced levels of Akkermansia and L. murinus, two famous species relevant to NAFLD and other metabolic disorders. In addition, ingestion of L. acidophilus, L. fermentum, L. paracasei, and L. plantarum markedly lowered the serum levels of triglyceride and total cholesterol of NAFLD mice, and consequently ameliorated the disease progression of NAFLD. According to the clinical pathology analysis, different Lactobacillus strains all significantly improved hepatic steatosis scores compared with western diets alone. Indeed, this study reinforces the protective effects of probiotics on NAFLD from the perspectives of both preclinical study and clinical practice, but more interestingly, it reminds us that the diagnosis and treatment of NAFLD could be together refined. Instead of being limited to the liver, a comprehensive understanding towards the metabolic profile of NAFLD and inter-organ crosstalk may help broaden the horizon.

Recently, an international expert group has announced the consensus of renaming NAFLD as metabolic (dysfunction) associated fatty liver disease (MAFLD), in two position papers. ${ }^{17}$.18 Although there is still some feeling in academia that the new acronym is premature, the effort to bring this disease to a more practical status and closer to metabolic disorders is undoubtedly destined. The new terminology and corresponding diagnostic criteria explicitly highlight overweight/obesity, type 2 diabetes mellitus, and metabolic dysregulation, which should bring more targets for mechanism research and intervention. Back to what we have discussed, the majority of animal studies and meta-analysis of clinical observations also support use of probiotics in treatment of obesity and metabolic disorders, for inducing weight loss and averting low- grade systemic inflammation, although these effects are still marginal compared with rigorous lifestyle modification. ${ }^{19,20}$ Application of probiotics in patients with MAFLD may have a bright prospect, considering good safety and tolerability profile shown in available enormous studies. However, the efficacy still needs to be validated by extensive studies with better design. Our limited knowledge regarding probiotics regimen, interventional timing and treatment duration at the moment, necessitates deeper understanding of how gut microbiota influences MAFLD pathogenesis. Further identification of the genetic and metabolic factors conferring the responsiveness of probiotics, as well as advanced detection means, will boost the implementation of personalized probiotic therapy in patients with MAFLD as well.

\section{Authors' contributions}

TY Ren: manuscript writing and critical revision; XY Li: manuscription revision; JG Fan: critical revision and supervision

\section{Acknowledgements}

The National Key Research and Development Program of China (No. 2017YFC0908903), National Natural Science Foundation of China (No. 81873565).

\section{Conflicts of Interest}

The authors have no conflicts to disclose.

\section{REFERENCES}

1. Fan JG, Kim SU, Wong VW. New trends on obesity and NAFLD in Asia. J Hepatol 2017;67:862-873.

2. Friedman SL, Neuschwander-Tetri BA, Rinella M, Sanyal AJ. Mechanisms of NAFLD development and therapeutic strategies. Nat Med 2018:24:908-922.

3. Oh H, Jun DW, Saeed WK, Nguyen MH. Non-alcoholic fatty liver diseases: update on the challenge of diagnosis and treatment. Clin Mol Hepatol 2016;22:327-335.

4. Byrne CD, Targher G. NAFLD: a multisystem disease. J Hepatol 2015;62(1 Suppl):S47-S64.

5. Zhang $X$, Ji X, Wang Q, Li JZ. New insight into inter-organ crosstalk contributing to the pathogenesis of non-alcoholic fatty liver disease (NAFLD). Protein Cell 2018;9:164-177.

6. Zhou D, Fan JG. Microbial metabolites in non-alcoholic fatty liver disease. World J Gastroenterol 2019:25:2019-2028.

7. Canfora EE, Meex RCR, Venema K, Blaak EE. Gut microbial metabolites in obesity, NAFLD and T2DM. Nat Rev Endocrinol 2019;15:261- 


\section{CLINCAL and MOLECULAR}

273.

8. Jena PK, Sheng L, Nagar N, Wu C, Barile D, Mills DA, et al. Synbiotics Bifidobacterium infantis and milk oligosaccharides are effective in reversing cancer-prone nonalcoholic steatohepatitis using western diet-fed FXR knockout mouse models. J Nutr Biochem 2018;57:246254.

9. Park SS, Lee YJ, Song S, Kim B, Kang H, Oh S, et al. Lactobacillus acidophilus NS1 attenuates diet-induced obesity and fatty liver. J Endocrinol 2018;237:87-100.

10. Kim B, Kwon J, Kim MS, Park H, Ji Y, Holzapfel W, et al. Protective effects of Bacillus probiotics against high-fat diet-induced metabolic disorders in mice. PLoS One 2018;13:e0210120.

11. Xiao MW, Lin SX, Shen ZH, Luo WW, Wang XY. Systematic review with meta-analysis: the effects of probiotics in nonalcoholic fatty liver disease. Gastroenterol Res Pract 2019;2019:1484598.

12. Duseja A, Acharya SK, Mehta M, Chhabra S, Shalimar, Rana S, et al. High potency multistrain probiotic improves liver histology in nonalcoholic fatty liver disease (NAFLD): a randomised, double-blind, proof of concept study. BMJ Open Gastroenterol 2019;6:e000315.

13. Famouri F, Shariat Z, Hashemipour M, Keikha M, Kelishadi R. Effects of probiotics on nonalcoholic fatty liver disease in obese children and adolescents. J Pediatr Gastroenterol Nutr 2017;64:413-417.

14. Shavakhi A, Minakari M, Firouzian $H$, Assali R, Hekmatdoost A, Ferns $G$. Effect of a probiotic and metformin on liver aminotrans- ferases in non-alcoholic steatohepatitis: a double blind randomized clinical trial. Int J Prev Med 2013;4:531-537.

15. Ahn SB, Jun DW, Kang BK, Lim JH, Lim S, Chung MJ. Randomized, double-blind, placebo-controlled study of a multispecies probiotic mixture in nonalcoholic fatty liver disease. Sci Rep 2019;9:5688.

16. Lee NY, Shin MJ, Youn GS, Yoon SJ, Choi YR, Kim HS, et al. Lactobacillus attenuates progression of nonalcoholic fatty liver disease by lowering cholesterol and steatosis. Clin Mol Hepatol 2021;27:110124.

17. Eslam M, Sanyal AJ, George J; International Consensus Panel. MAFLD: a consensus-driven proposed nomenclature for metabolic associated fatty liver disease. Gastroenterology 2020;158:19992014.e1.

18. Eslam M, Newsome PN, Sarin SK, Anstee QM, Targher G, RomeroGomez $M$, et al. A new definition for metabolic dysfunctionassociated fatty liver disease: an international expert consensus statement. J Hepatol 2020;73:202-209.

19. Vallianou N, Stratigou T, Christodoulatos GS, Tsigalou C, Dalamaga M. Probiotics, prebiotics, synbiotics, postbiotics, and obesity: current evidence, controversies, and perspectives. Curr Obes Rep 2020;9:179-192.

20. Tenorio-Jiménez C, Martínez-Ramírez MJ, Gil Á, Gómez-Llorente C. Effects of probiotics on metabolic syndrome: a systematic review of randomized clinical trials. Nutrients 2020;12:124. 